\title{
Mode Coupling in Plasmonic Heterodimers Probed with Electron Energy Loss Spectroscopy
}

\author{
Valentin Flauraud, ${ }^{\dagger}{ }^{\dagger}$ Gabriel D. Bernasconi, ${ }^{\ddagger}$ Jérémy Butet, ${ }^{\ddagger \odot}$ Duncan T. L. Alexander, ${ }^{\S}$
} Olivier J. F. Martin, ${ }^{*}$ and Jürgen Brugger*, ${ }^{\dagger}$

${ }^{\dagger}$ Microsystems Laboratory, Institute of Microtechnique, École Polytechnique Fédérale de Lausanne, 1015 Lausanne, Switzerland

${ }^{\ddagger}$ Nanophotonics and Metrology Laboratory, École Polytechnique Fédérale de Lausanne, 1015 Lausanne, Switzerland

${ }^{\S}$ Interdisciplinary Centre for Electron Microscopy (CIME), École Polytechnique Fédérale de Lausanne, 1015 Lausanne, Switzerland

Supporting Information

ABSTRACT: While plasmonic antennas composed of building blocks made of the same material have been thoroughly studied, recent investigations have highlighted the unique opportunities enabled by making compositionally asymmetric plasmonic systems. So far, mainly heterostructures composed of nanospheres and nanodiscs have been investigated, revealing opportunities for the design of Fano resonant nanostructures, directional scattering, sensing and catalytic applications. In this article, an improved fabrication method is reported that enables precise tuning of the heterodimer geometry, with interparticle distances made down to a few nanometers between $\mathrm{Au}-\mathrm{Ag}$ and $\mathrm{Au}-\mathrm{Al}$ nanoparticles. $\mathrm{A}$

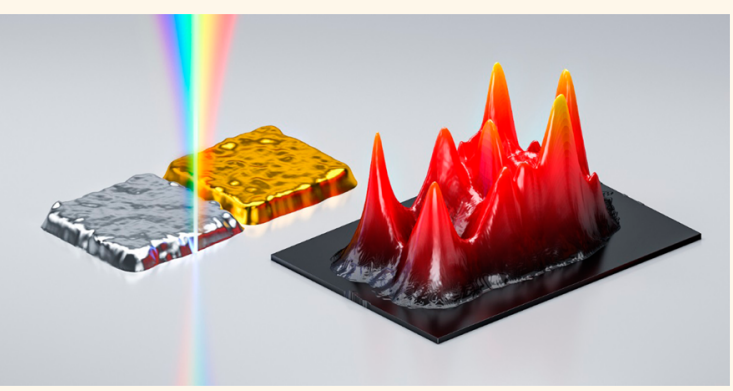
wide range of mode energy detuning and coupling conditions are observed by near field hyperspectral imaging performed with electron energy loss spectroscopy, supported by full wave analysis numerical simulations. These results provide direct insights into the mode hybridization of plasmonic heterodimers, pointing out the influence of each dimer constituent in the overall electromagnetic response. By relating the coupling of nondipolar modes and plasmon-interband interaction with the dimer geometry, this work facilitates the development of plasmonic heterostructures with tailored responses, beyond the possibilities offered by homodimers.

KEYWORDS: optical nanoantennas, plasmonic heterostructures, bimetallic antennas, electron energy loss spectroscopy, electron beam lithography, eigenmodes

ollective oscillations of the conduction electrons in metal nanostructures, known as localized surface plasmon resonances, ${ }^{1}$ have been intensively studied and designed by manipulating both the nanostructures size and geometry, as well as their dielectric environment. ${ }^{2}$ These investigations, carried out over the entire visible light spectrum, including the near-UV and near-IR, have demonstrated the control of both nanoscale optical fields and far field radiation, ${ }^{1}$ leading to the concept of optical nanoantennas. ${ }^{2,3}$ The electromagnetic properties of these structures are governed by their eigenmodes, ranging from dipoles to high order multipoles. ${ }^{4,5}$ When the nanoparticles are arranged in pairs or multimers, these modes couple to each other and hybridize, producing further optical properties. ${ }^{6-8}$ In the simplest and most common geometry, involving the coupling of two selfsimilar spherical nanoparticles separated by a nanogap, a wellknown dipole-dipole interaction along the dimer axis is produced..$^{6}$ This coupling generates an intense and confined near field in the gap region, which can enable large nanoscale fluorescence enhancement ${ }^{9}$ and surface enhanced Raman scattering down to the single molecule level. ${ }^{10,11}$ Thanks to a complete description of the plasmonic coupling for spherical self-similar nanoparticle dimers, Nordlander and coauthors ${ }^{7}$ have shown that the gap dependent hybridization in such nanostructures stems from the interaction of the uncoupled eigenmodes. Specifically, the uncoupled modes hybridize with bonding and antibonding interactions and each of these two configurations occur along the longitudinal and transverse axes of the nanodimer. As gap size decreases, and hence mode coupling increases, the bonding modes exhibit large red-shifts, whereas antibonding modes are subject to moderate blue-shifts. Additionally, the relative phase of the charge oscillation between the two nanoparticles may lead to either enhanced

Received: December 22, 2016

Accepted: March 14, 2017

Published: March 14, 2017 

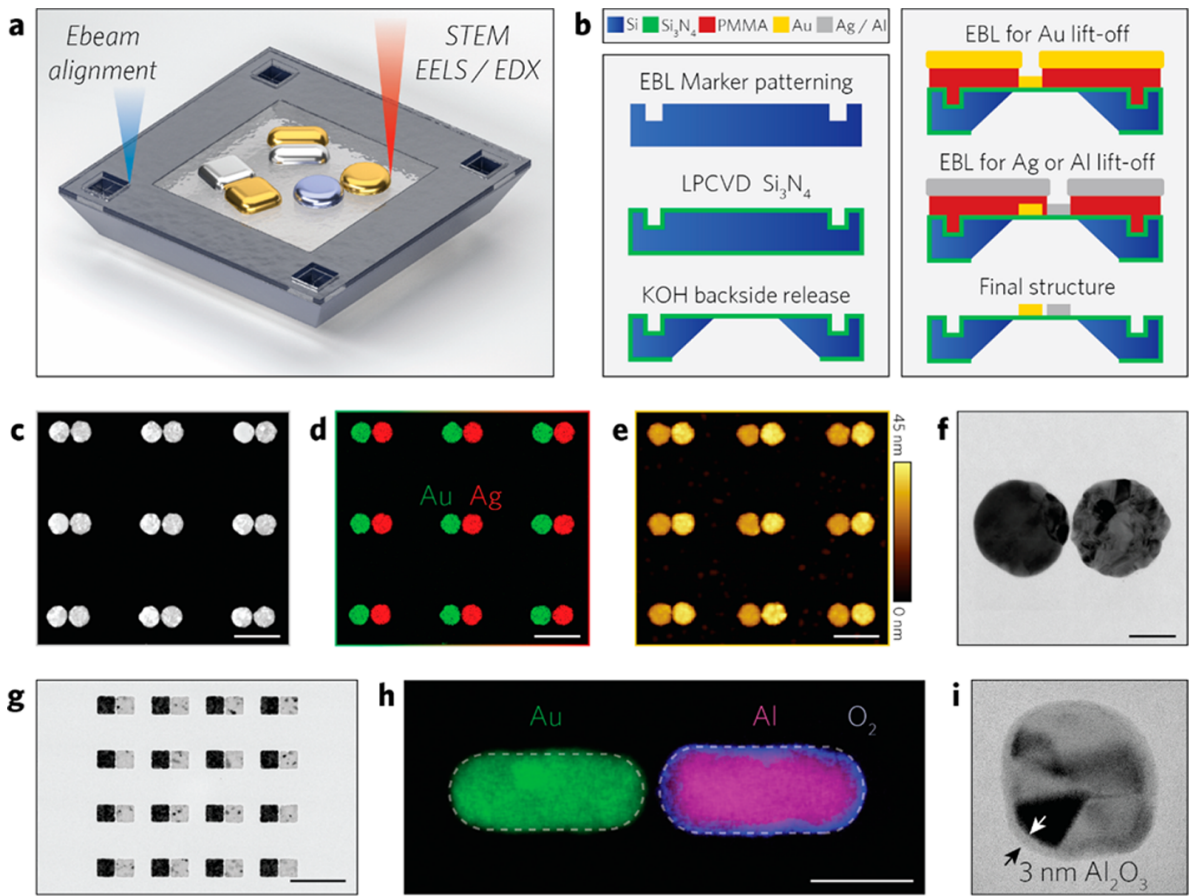

Figure 1. Heterodimer fabrication. (a) Schematic representation of a freestanding $\mathrm{Si}_{3} \mathrm{~N}_{4}$ membrane, lithographically patterned structures, and electron beam lithography alignment marks on the $\mathrm{Si}$ frame. (b) Fabrication process including marker patterning, membrane release, and subsequent aligned lift-off steps. (c) HAADF STEM image of Au-Ag dimers, (d) corresponding EDX Au-Ag map, and (e) AFM topography map. (f) TEM image of a single, nanometer gap dimer. (g) Au-Al square dimer array. (h) Au-Al nanorod dimer EDX map, with the Al surface oxide shown in blue. (i) TEM image of the $\mathrm{Al}_{2} \mathrm{O}_{3}$ layer, shown by a lack of diffraction contrast in comparison with the crystalline $\mathrm{Al}$ grain. Scale bars are $200 \mathrm{~nm}(\mathrm{c}, \mathrm{d}, \mathrm{e}), 50 \mathrm{~nm}(\mathrm{f}, \mathrm{h})$, and $500 \mathrm{~nm}(\mathrm{~g})$, respectively.

or vanishing net dipole moments, so forming, respectively, bright and dark modes. ${ }^{12}$

Beyond symmetric dimers, heterogeneous pairs formed by two dissimilar nanoparticles, each having distinct spectral properties owing to a compositional or geometrical difference, have recently become a topic of wide interest. The resulting near field overlap and far field interference of eigenmodes with distinct energy or multipolar orders have been studied for a wealth of unique properties, such as directional scattering; ${ }^{13-15}$ Fano resonances in both radiation ${ }^{16-18}$ and absorption processes; ${ }^{19,20}$ enhanced nonlinear processes; ${ }^{21}$ photothermal effects; ${ }^{22}$ sensing; ${ }^{15,23-25}$ and catalysis. ${ }^{26,27}$ As with symmetric dimers, the hybridized eigenmodes of these heterogeneous pairs (known as heterodimers) also originate from the uncoupled eigenmodes supported by each dimer constituent. $^{7,16,28}$ However, due to the reduced symmetry of the system, the hybridized modes of heterodimers will be less likely to possess a vanishing dipole moment, the latter effect being a direct consequence of the mirror symmetry of homodimers.

To date, most experimental studies of heterodimers have focused on their far field properties, by monitoring their dark field spectra ${ }^{16,29,30}$ and radiation patterns. ${ }^{14}$ Here we instead evidence the benefit of direct experimental investigations of their modal responses, especially for geometries beyond the sphere and disc dimers. Indeed, only a few experimental studies, mostly limited to large nanostructures exhibiting minimal energy mismatch ${ }^{31}$ and limited design flexibility, ${ }^{32,33}$ have focused on measuring the near field properties of heterodimers in order to visualize and tailor their electromagnetic properties. In this article, we reveal the coupled modes of a wide range of heterodimers using electron energy loss spectroscopy (EELS) combined with a full wave analysis based on surface integral equations (SIE) methods. By fabricating heterodimers of different materials and geometries using a precise multilayer electron beam lithography fabrication process, we span a large range of energy detuning conditions and coupling strengths between each dimer component, thereby unveiling the rich electromagnetic properties of mismatched particle pairs.

\section{RESULTS AND DISCUSSION}

Motivated by the unique opportunities enabled by asymmetric plasmonic systems, a vast palette of enabling nanofabrication methods has been developed. Exploiting the mode energy mismatch in heterodimers calls for techniques that can create narrow interparticle gaps in order to significantly hybridize the eigenmodes of the constituent elements. ${ }^{16}$ To this end, colloidal assembly methods have been effective for providing simple access to few nanometer gaps, with techniques varying from capillary assembly ${ }^{34,35}$ and electrostatic interactions $^{16,19,24,36}$ to DNA linkers ${ }^{29,37}$ and origamis. ${ }^{30,38}$ A different approach relies on the use of dielectric microbeads for holesphere lithography schemes, combined with tilted evaporation. ${ }^{14,15,33,39-42}$ Although these various techniques are highly scalable and cost efficient, they suffer from a poor geometric degree of freedom. While mode energy detuning is the central aspect of asymmetric plasmonic systems, the mediation of coupling strength by controlling the geometry of each nanoparticle and their gap distance is essential to tailor their electromagnetic response. To this end, compositional asymmetry achieved with multistep lithography can be used advantageously to disentangle the influence of the mode energies and nanoparticle shapes on the coupled modes. To realize this capability, a double layer lift-off process based on electron beam lithography ${ }^{21,25}$ was developed and optimized in 


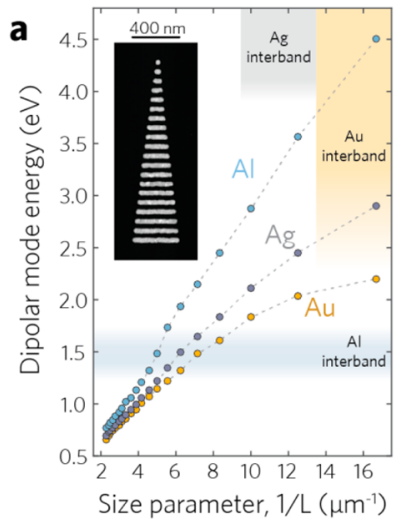

b

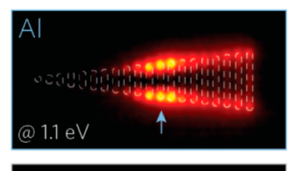

C

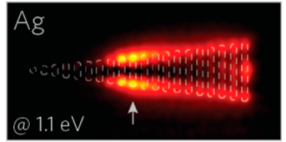

d

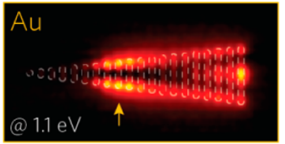

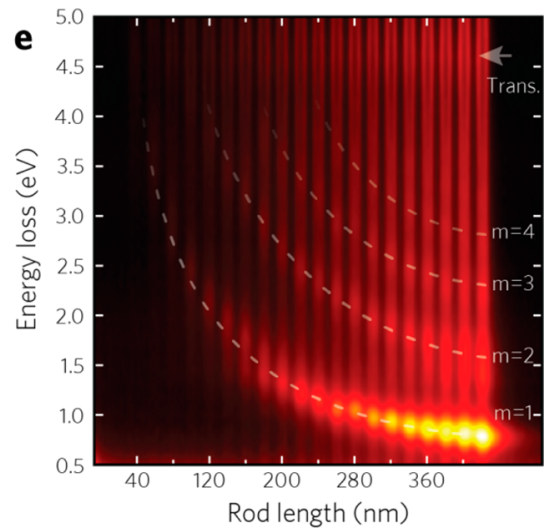

Figure 2. Material dependent resonance energies. (a) Resonant energy of the dipole mode as a function of the inverse of the nanoparticle length $L$, measured from a series of nanoparticles of varying aspect ratios as shown in the HAADF STEM image in inset. Interband regions are shown schematically. (b-d) EELS intensity maps extracted at $1.1 \mathrm{eV}$ from the three datacubes acquired for similar parallel nanorod sequences made with $\mathrm{Al}, \mathrm{Ag}$, and $\mathrm{Au}$, respectively. (e) EELS response from the $\mathrm{Al}$ nanorod sequence. The datacube was binned spatially along the nanorod axis yielding an energy against rod length visualization, displayed here to highlight the size dependent evolution of higher order modes in Al nanorods.

a

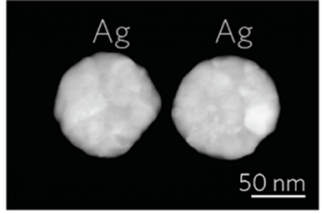

b

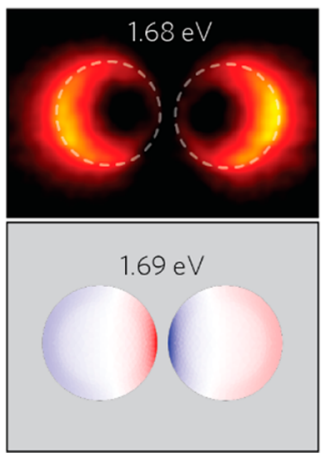

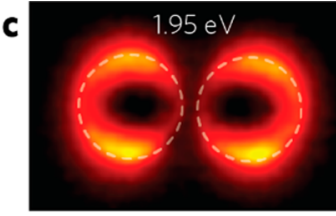

d

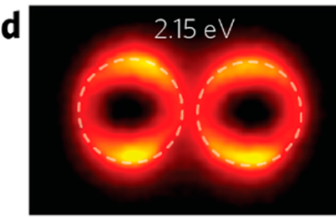

e

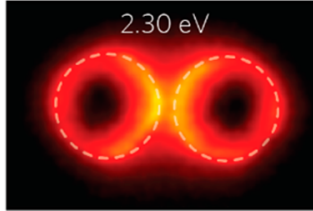

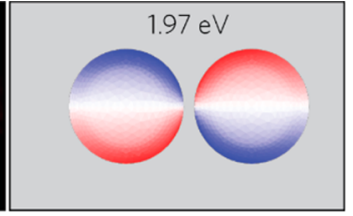
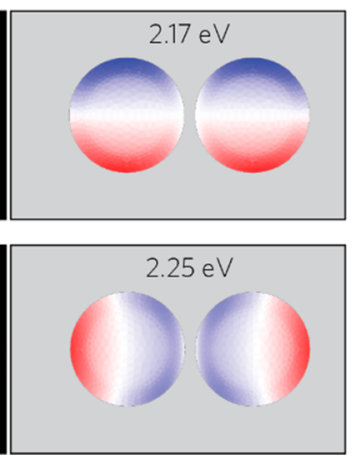
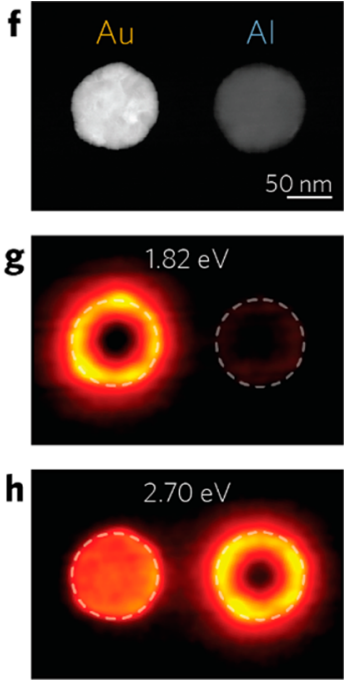

Figure 3. EELS response of a coupled homodimer and weakly coupled heterodimer. (a) HAADF STEM image of an Ag homodimer with $\sim 100$ $\mathrm{nm}$ disc diameter and $\sim 9 \mathrm{~nm}$ gap width. Experimental EELS maps of the homodimer and associated computed eigencharge distributions for (b) the longitudinal bonding, (c) the transverse bonding, (d) the transverse antibonding, and (e) the longitudinal antibonding modes. (f) AuAl heterodimer with $\sim 100 \mathrm{~nm}$ disc diameter and $60 \mathrm{~nm}$ gap width and the associated EELS intensity maps of the dipole modes of (g) the Au nanoparticle and (h) the $\mathrm{Al}$ nanoparticle.

order to reach few nanometer gap distances while exploiting the geometric freedom of lithography. The process, outlined in Figure $1 \mathrm{a}, \mathrm{b}$, is entirely carried out on freestanding $\mathrm{Si}_{3} \mathrm{~N}_{4}$ membranes; an approach that enables high resolution metrology of the heterodimers by transmission electron microscopy as well as their electromagnetic characterization with EELS. Briefly, alignment marks are etched on the front side of a silicon substrate, which is subsequently coated with low-pressure chemical vapor deposition $\mathrm{Si}_{3} \mathrm{~N}_{4}$. The $\mathrm{Si}_{3} \mathrm{~N}_{4}$ layer is then locally released by backside processing. Next, multiple steps of electron beam lithography, evaporation and lift-off steps are repeated on the membrane for each $\mathrm{Au}, \mathrm{Ag}$ and $\mathrm{Al}$ layer (see Methods for details). The high quality markers and the related alignment scheme reliably enable a sub-10 nm layerto-layer alignment accuracy and a reproducible fabrication of heterodimers arrays with few-nanometer interparticle distances. The ultimate nanogap resolution and dispersion are limited by the metal line-edge roughness and the associated thin-film polycrystallinity (Figure S1). After fabrication, structure topography was characterized by atomic force microscopy, and the overall dimensions and gaps were measured by transmission electron microscopy and high angle annular dark field scanning transmission electron microscopy (HAADF $\mathrm{STEM}$ ), for both $\mathrm{Au}-\mathrm{Ag}$ (Figure $1 \mathrm{c}-\mathrm{f}$ ) and $\mathrm{Au}-\mathrm{Al}$ heterodimers (Figure $1 \mathrm{~g}-\mathrm{i}$ ). In addition, scanning transmission electron microscopy energy dispersive X-ray spectroscopy (EDX) was performed to verify the quality and absence of contamination in the Ag nanostructures (Figure S2), as well as the composition and stability of the $3 \mathrm{~nm}$-thick alumina layer on the Al nanoparticle surfaces.

$\mathrm{Au}, \mathrm{Ag}$ and $\mathrm{Al}$ were chosen as prototypical materials of the constituent nanoparticles due to their ability to support plasmonic resonances and interband transitions at distinct energies. While layered ${ }^{43}$ and alloyed ${ }^{44}$ materials may also be of interest for the fabrication of heterogeneous plasmonic systems, the present study focuses on the coupling of the aforemen- 

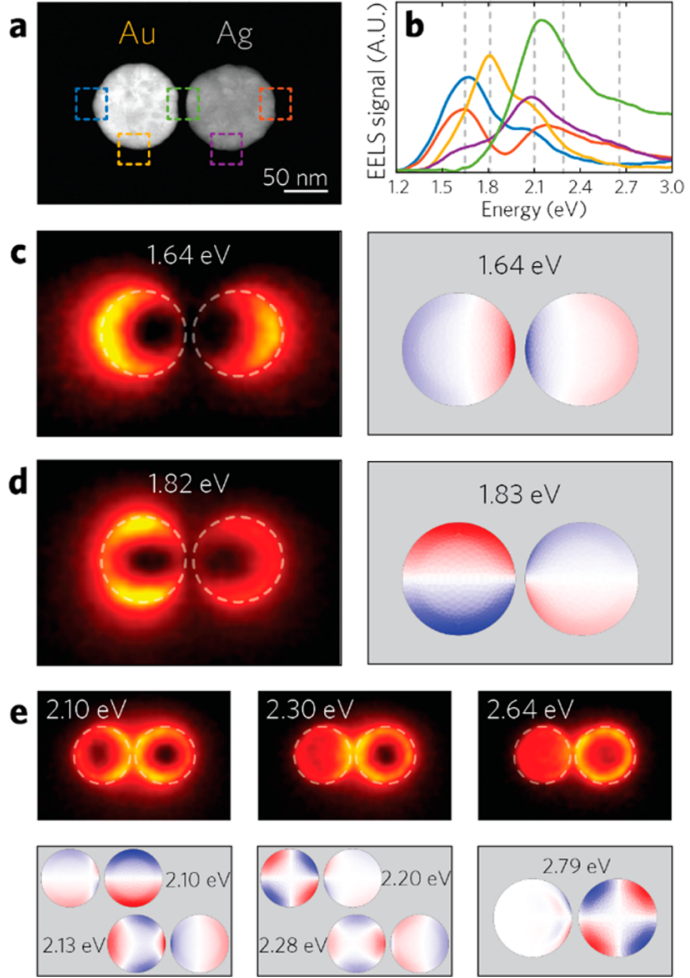
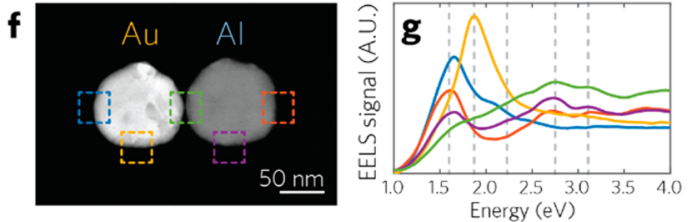

h
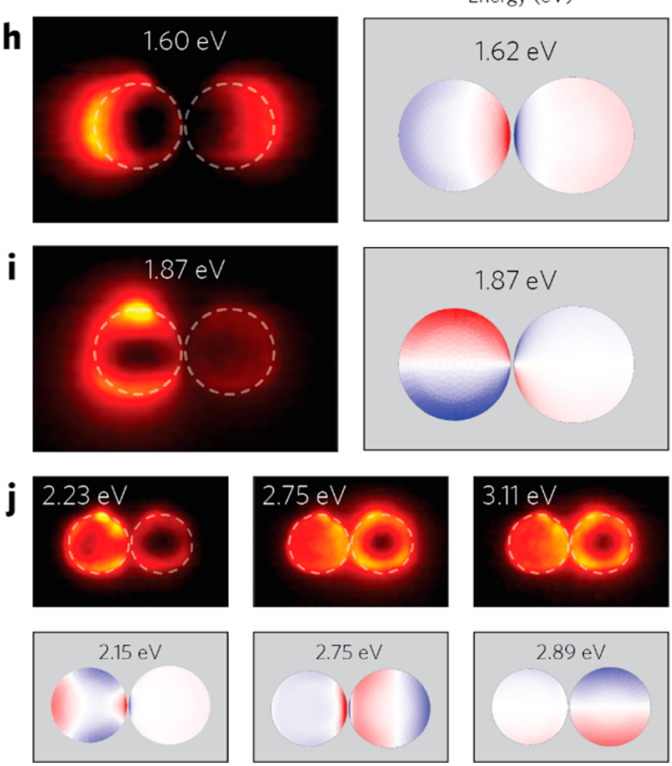

Figure 4. Coupled Au-Ag and Au-Al disc heterodimers. (a) HAADF STEM image of an Au-Ag heterodimer with $\sim 100$ nm disc diameter and $\sim 10 \mathrm{~nm}$ gap width. (b) Experimental EELS spectra corresponding to the impact positions highlighted in (a). EELS maps and associated eigencharges for modes dominated by (c) the longitudinal dipole mode in the Au nanoparticle, (d) the transverse dipole mode in the Au nanoparticle, and (e) some combinations of dipole mode in the Ag nanoparticle with high order modes of the Au nanoparticle. (f) HAADF STEM image of an $\mathrm{Au}-\mathrm{Al}$ heterodimer with $\sim 100 \mathrm{~nm}$ disc diameter and $\sim 2 \mathrm{~nm}$ gap width. (g) Experimental EELS spectra corresponding to the impact positions highlighted in (f). EELS maps and associated eigencharges for modes dominated by (h) the longitudinal dipole mode in the Au nanoparticle, (i) the transverse dipole mode in the Au nanoparticle, and (j) some combinations of dipole mode in the Al nanoparticle with high order modes of the Au nanoparticle (j).

tioned pure metals. ${ }^{45,46}$ In order to first characterize the response of each constituent metal used to make the heterodimers, EELS measurements were performed on monometallic $\mathrm{Au}, \mathrm{Ag}$ and $\mathrm{Al}$ arrays of nanorods with varying lengths, ranging from a $40 \mathrm{~nm}$ diameter dot to a $400 \times 40 \mathrm{~nm}$ rod. By measuring the evolution of the longitudinal dipolar mode energy as a function of the nanorod size, the spectral tunability and resonance range of the different materials is evidenced for this specific morphology evolution (Figure 2a). Resonance energy differences between each material stem from their different free electron densities and associated plasma frequencies as well as the difference in their intrinsic losses due to collisions. As particle aspect ratio decreases (and wavenumber increases), the energy difference between dipole modes of two equivalently sized nanoparticles of different materials increases. Al displays the largest energy tunability across the geometric parameter space investigated here, ${ }^{47}$ due to its higher plasma frequency. ${ }^{48}$ Interestingly, the interband transitions of $\mathrm{Al}$, occurring at $1.5 \mathrm{eV}$, are well evidenced by a discontinuity in the dispersion curve around this energy loss. Already for EELS intensity maps extracted at a relatively low energy $(1.1 \mathrm{eV}$ Figure $2 b-d)$, the detuning between the different materials is clearly observed, as their dipolar modes are supported by nanorods of different lengths; a quadrupolar EELS response also appears on the longest $\mathrm{Au}$ and $\mathrm{Ag}$ nanorods. Note that the localization and symmetry of the focused electron probe used in STEM EELS allows for an efficient excitation of high order modes, regardless of their radiative nature ${ }^{49}$ as shown in Figure 2e. Within the parameter space displayed, it is clear that bimetallic dimers formed from nanoparticles of equal dimensions may exhibit two distinct mode energy mismatch conditions. First, for large nanostructures, the dipolar and/or higher order modes of one nanoparticle may spectrally overlap with those of the other, resulting in a significant plasmonic coupling. Second, a different condition is predicted for small particle dimers, especially for $\mathrm{Au}-\mathrm{Al}$ pairings, where the energy of the fundamental dipole of the Al component may be at sufficiently high energy to avoid having any significant spectral overlap with any of the modes supported by the $\mathrm{Au}$ component. Both of these coupling conditions are investigated in the next section, by the study of the near field response of selected heterodimers.

First we highlight the specificities of EELS signals associated with mode hybridization in symmetric pairs, ${ }^{50-52}$ by investigating the response of an $\mathrm{Ag}-\mathrm{Ag}$ disc homodimer with a diameter of $100 \mathrm{~nm}$ and gap of $9 \mathrm{~nm}$ (Figure 3a). The eigenmodes of the corresponding structure are computed using a surface integral equation (SIE) method, solving for the eigenvectors of the associated matrix. ${ }^{53}$ Four modes, mainly based on dipole-dipole interactions, are identified, and compared to those of an isolated $\mathrm{Ag}$ monomer of similar dimensions having a dipole resonance energy of $\sim 2.12 \mathrm{eV}$ (Figure S3). As expected, the bonding modes (longitudinal and transverse Figure $3 b, c)$ are red-shifted in comparison to the dipolar mode supported by the solitary Ag disc. The strong coupling of the bonding longitudinal mode (Figure $3 \mathrm{~b}$ ) is 
evidenced by a larger red-shift, along with a strong EELS intensity inhomogeneity in each nanoparticle which vanishes in the gap region. This insensitivity of EELS to bonding modes in gap regions, as compared to strong gap sensitivity to antibonding modes, has been thoroughly discussed in relation to the field symmetry of the electron probe. ${ }^{54,55}$ Although EELS does not directly map the electromagnetic near field and cannot reveal high intensity hot-spots formed by bonding interactions, this apparent limitation actually proves useful in distinguishing the coupling nature and symmetry of hybridized modes. The aforementioned signal silencing in the gap is also seen for the transverse bonding modes in Figure 3c, but to a lesser extent due to the weak interaction between transverse modes. In contrast, both of the higher energy transverse (Figure 3d) and longitudinal modes (Figure 3e) exhibit a strong EELS signal in the gap region, which expresses their antibonding nature. In agreement with the simulated eigenmodes, the longitudinal antibonding mode is the most blue-shifted, owing to a strong near field interaction for this dipole orientation. Besides small asymmetries in the EELS intensity maps, which arise from the grain structure of the fabricated nanodimers, the near field maps of the two dimers have a mirror symmetry, in keeping with their compositional and structural symmetry.

The EELS response of a heterodimer is now considered, but one with limited coupling. In order to minimize the electromagnetic interactions, discs made of $\mathrm{Au}$ and $\mathrm{Al}$ are chosen, yielding a large energy separation between their isolated particle plasmonic modes. They are positioned 60 $\mathrm{nm}$ apart from each other (Figure 3f). In consequence, each disc's unique dipolar mode is observed, at $1.82 \mathrm{eV}$ (Figure $3 \mathrm{~g}$ ) and $2.70 \mathrm{eV}$ (Figure $3 \mathrm{~h}$ ) for $\mathrm{Au}$ and $\mathrm{Al}$, respectively. Due to the weak coupling between the nanoparticles, the longitudinal and transverse modes do not split and are therefore degenerated. ${ }^{56}$ Consistent with this, the EELS maps display no nodes or specific symmetry relations to the dimer axis. At $2.70 \mathrm{eV}$ the $\mathrm{Au}$ nanoparticle shows a strong and uniform EELS signal, corresponding to uniform interband absorption inside it (Figure 2a).

Now we move to a heterodimer displaying strong mode hybridization, by investigating an $\mathrm{Au}-\mathrm{Ag}$ disc dimer (Figure $4 \mathrm{a}$ ) with the same geometry as that of the $\mathrm{Ag}-\mathrm{Ag}$ dimer discussed earlier. The EELS spectra extracted at different locations of this heterodimer (Figure 4b) show various resonances, the intensity of which varies with the position of the electron beam, as also observed in EELS simulations (Figure S4). The EELS maps suggest that the electromagnetic near field is mainly localized close to the Au nanoparticle for the two lowest energy modes (that support a dominant longitudinal (Figure 4c) and transverse (Figure 4d) dipolar contribution). This is in agreement with previous EELS computations performed for detuned dimers. ${ }^{57}$ As revealed by the eigenmode simulations, the charge distributions on the Ag disc surface (Figure 4c,d) also have a dipolar nature, although weaker and with opposite phase. This behavior is due to the energy mismatch of the dipolar modes of each nanoparticle and is reminiscent of the transverse antibonding mode of a symmetric structure (Figure 3c). In this $\mathrm{Au}-\mathrm{Ag}$ heterostructure, the overlap between the Ag dipole $(\sim 2.12 \mathrm{eV})$ and $\mathrm{Au}$ quadrupole $(\sim 2.22 \mathrm{eV}$ Figure S3) allows for a strong interaction between these modes (Figure 4e). This especially holds for the longitudinal hybridized modes, as also evidenced in simulations, that tend to bonding (eigenmode at $2.13 \mathrm{eV}$ ) and nearly antibonding (eigenmode at $2.28 \mathrm{eV}$ ) configurations, reminiscent of observations made in symmetric dimers (Figure $3 \mathrm{~b}, \mathrm{e})$. As discussed previously, the weaker interaction between transverse modes leads to two hybridized modes with a limited coupling (and thus energy shift), one with a strong transverse dipole mode in the $\mathrm{Ag}$ nanoparticle (eigenmode at $2.10 \mathrm{eV}$ ) and another with a strong transverse quadrupole mode in the $\mathrm{Au}$ nanoparticle (eigenmode at $2.20 \mathrm{eV}$ ). Here we use the terminology transverse quadrupole for modes having an odd parity (charge-wise) relatively to the dimer axis, and longitudinal for an even parity, analogously to the dipole modes. The spectral proximity of these four modes, between 2.10 and $2.28 \mathrm{eV}$, results in a partial overlap of their contribution in the experimental EELS maps. Nevertheless, the nature of the dominant longitudinal modes is evident from the subtle differences in the EELS intensity maps such as the stronger signal confinement in the gap region at $2.30 \mathrm{eV}$ from the antibonding mode compared to the bonding mode at 2.10 $\mathrm{eV}$. For the quadrupole mode in the Ag nanoparticle, the EELS map $(2.64 \mathrm{eV})$ shows a rotational symmetry around the center of the Ag disc, indicating that the coupling of this mode with the nearby Au disc is weak. This is in agreement with the calculated eigenmode that shows a charge distribution on the Ag disc barely affected by the neighboring Au disc. Overall, our analysis provides an important insight into the response of this system which has been previously studied for the purpose of directional color routing. ${ }^{14}$ Namely, all the observed modes possess a net dipole moment and therefore varying degree of far-field radiation symmetry (Figure S5). When the coupled modes entail a large energy mismatch, they are predominantly supported by a single constituent (here the dipole in the $\mathrm{Au}$ nanodisc); whereas a collective response is favored for modes with matching energy (here the quadrupole in the Au disc and the dipole in the $\mathrm{Ag}$ one), even if the original modes have a distinct multipolar nature.

A similar analysis was carried out for smaller dimers with 50 $\mathrm{nm}$ disc diameter (Figure S6), demonstrating the influence of the dimer size, associated with energy tuning, on the mode hybridization. In the case of small $\mathrm{Au}-\mathrm{Ag}$ discs, the increased energy mismatch between the $\mathrm{Au}$ and $\mathrm{Ag}$ modes (Figure 2) leads to a minimal contribution of the high order (i.e., quadrupole) modes. Indeed, only the longitudinal dipole mode of the $\mathrm{Au}$ nanoparticle exhibits a significant coupling with the Ag nanoparticle (Figure S6c). The intrinsic modes of the $\mathrm{Ag}$ nanoparticle are at energies corresponding to the $\mathrm{Au}$ interband transitions, and so are only weakly coupled to the $\mathrm{Au}$ nanoparticle. At this point, the Au disc effectively acts as a modification of the dielectric environment of the $\mathrm{Ag}$ nanoparticle with nonresonant properties (Figure S6e-g).

These observations on the $50 \mathrm{~nm}$ disc diameter $\mathrm{Au}-\mathrm{Ag}$ heterodimer, which are in excellent agreement with previous far-field optical studies of geometrically comparable systems, ${ }^{29,30}$ prompt the investigation of phenomena occurring with the large energy detuning associated with $\mathrm{Au}-\mathrm{Al}$ disc dimers (Figure $4 \mathrm{f}-\mathrm{j}$ ). The small gap width $(2 \mathrm{~nm})$ is intended to compensate for the large spectral mismatch between the modes of the isolated nanoparticles, by creating a strong near field overlap. Similarly to the $\mathrm{Au}-\mathrm{Ag} 100 \mathrm{~nm}$ disc dimer studied previously, EELS spectra were recorded for various positions of the electron beam (Figure $4 \mathrm{~g}$ ) and intensity maps made for different observed resonance energies (Figure $4 \mathrm{~h}-\mathrm{j}$ ). A near field asymmetry much larger than that for the $\mathrm{Au}-\mathrm{Ag}$ $100 \mathrm{~nm}$ disc dimer is revealed for the low energy maps, as the 

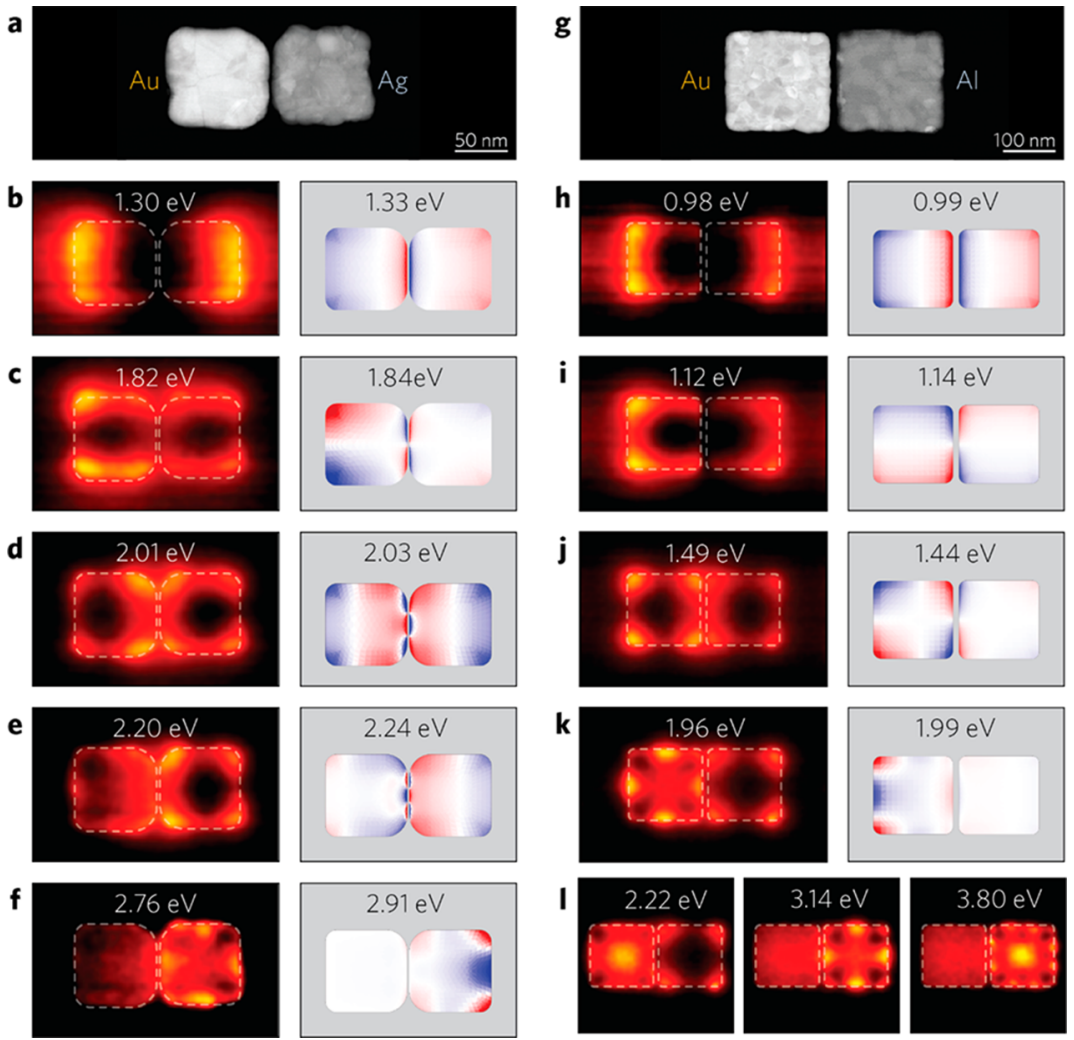

Figure 5. Coupled $\mathrm{Au}-\mathrm{Ag}$ and $\mathrm{Au}-\mathrm{Al}$ square heterodimers. (a) HAADF STEM image of a Au-Ag square heterodimer with $100 \mathrm{~nm}$ square edge length and $1.5 \mathrm{~nm}$ gap. (b-f) EELS maps and the corresponding computed eigencharge distributions for the Au-Ag dimer. (g) HAADF STEM image of a $\mathrm{Au}-\mathrm{Al}$ square heterodimer with $200 \mathrm{~nm}$ square edge length and $10 \mathrm{~nm}$ gap. (h-l) EELS maps and the corresponding computed eigencharge distributions for the $\mathrm{Au}-\mathrm{Al}$ dimer.

dipole in the Au nanodisc couples only weakly to the dipole in the $\mathrm{Al}$ disc in both the longitudinal (Figure $4 \mathrm{~h}$ ) and transverse (Figure 4i) directions. Also, for this structure, the near field coupling involving the longitudinal quadrupole in the $\mathrm{Au}$ nanodisc is now well evidenced because of its limited overlap with other modes (Figure 4j $2.23 \mathrm{eV}$ ). The EELS maps for the longitudinal dipole $(2.75 \mathrm{eV})$ and transverse dipole $(3.11 \mathrm{eV})$ in the $\mathrm{Al}$ nanodisc in turn exhibit limited coupling and thus rotational symmetry. These results are similar to the observations made for the $\mathrm{Au}-\mathrm{Ag}$ dimer with $50 \mathrm{~nm}$ disc diameter where, again, each coupled mode is mainly associated with one constituent of the dimer. Nevertheless, the EELS maps associated with the higher energy modes (Figure 4j) exhibit strong signal modulations in the Au disc. This evidences the strong absorption modulation in the off resonant system, since the near-field associated with the resonant component decays into the nonresonant nanoparticle. ${ }^{20,26}$

In order to favor the resonant interaction between detuned modes, we exploit the opportunities of our lithographic fabrication method to design square dimers. Compared to round dimers, these structures allow for a distinct Coulomb interaction at similar gap distances. These structures also break the rotational symmetry in discs that was observed with some EELS maps, while preserving the degeneracy of the uncoupled eigenmodes along the longitudinal and transverse directions. Considering first an $\mathrm{Au}-\mathrm{Ag}$ dimer with a $100 \mathrm{~nm}$ square side length and average $2.2 \mathrm{~nm}$ gap (Figure 5a), very limited asymmetry in the lowest energy mode is seen (Figure $5 b$ ) as compared to the disc case, due to the enhanced coupling. On the other hand, asymmetry in the EELS map is observed for a transverse dipole in the $\mathrm{Au}$ nanosquare, which is more weakly coupled (Figure 5c). Now considering higher order modes, due to the strong spatial coupling conditions induced by the gap geometry, a complex hybridization scheme is expected, involving multiple high order contributions. ${ }^{7}$ This is seen with the coupling of the longitudinal dipole in the $\mathrm{Ag}$ nanoparticle to highly multipolar modes in the Au nanoparticle (Figure 5d,e), where the eigencharge distributions for the closest energy matching modes reveal numerous nodes in the gap region. Above the $\mathrm{Au}$ interband threshold, the $2.76 \mathrm{eV}$ EELS map is characteristic of an octupole in the $\mathrm{Ag}$ nanoparticle (Figure 5f) with a limited coupling to the Au nanoparticle. Similar observations are made for a larger (200 $\mathrm{nm}$ square side length) $\mathrm{Au}-\mathrm{Al}$ dimer with $15 \mathrm{~nm}$ gap (Figure $5 g-1)$. Considering the dispersion curves in Figure 2a, the large square size allows for a reduced energy mismatch between the $\mathrm{Au}$ and $\mathrm{Al}$ nanoparticles compared to the $\mathrm{Au}-\mathrm{Al}$ disc dimer discussed previously. Additionally, the larger gap limits the contribution of high order modes that lead to complex nodal patterns in the gap. Low energy longitudinal (Figure 5h) and transverse (Figure 5i) modes follow the mechanisms pointed out previously; however the interplay between high order modes is better revealed in this differently coupled nanodimer. Quadrupole (Figure 5j) and octupole (three nodal lines) (Figure 5k) modes in the $\mathrm{Au}$ nanoparticle now couple efficiently to nondipolar modes in the $\mathrm{Al}$ one. Indeed, the quadrupolar mode in the Au nanoparticle is coupled to another weak quadrupolar charge distribution in the $\mathrm{Al}$ nanoparticle, whereas, at even higher energy, the Au octupolar mode couples weakly to the $\mathrm{Al}$ nanoparticle, leading to asymmetric surface 

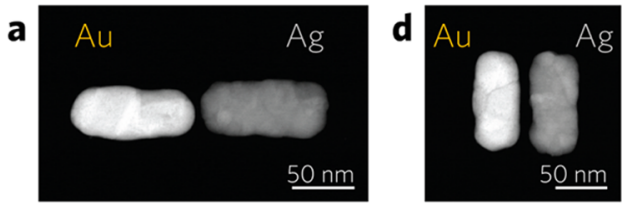

b
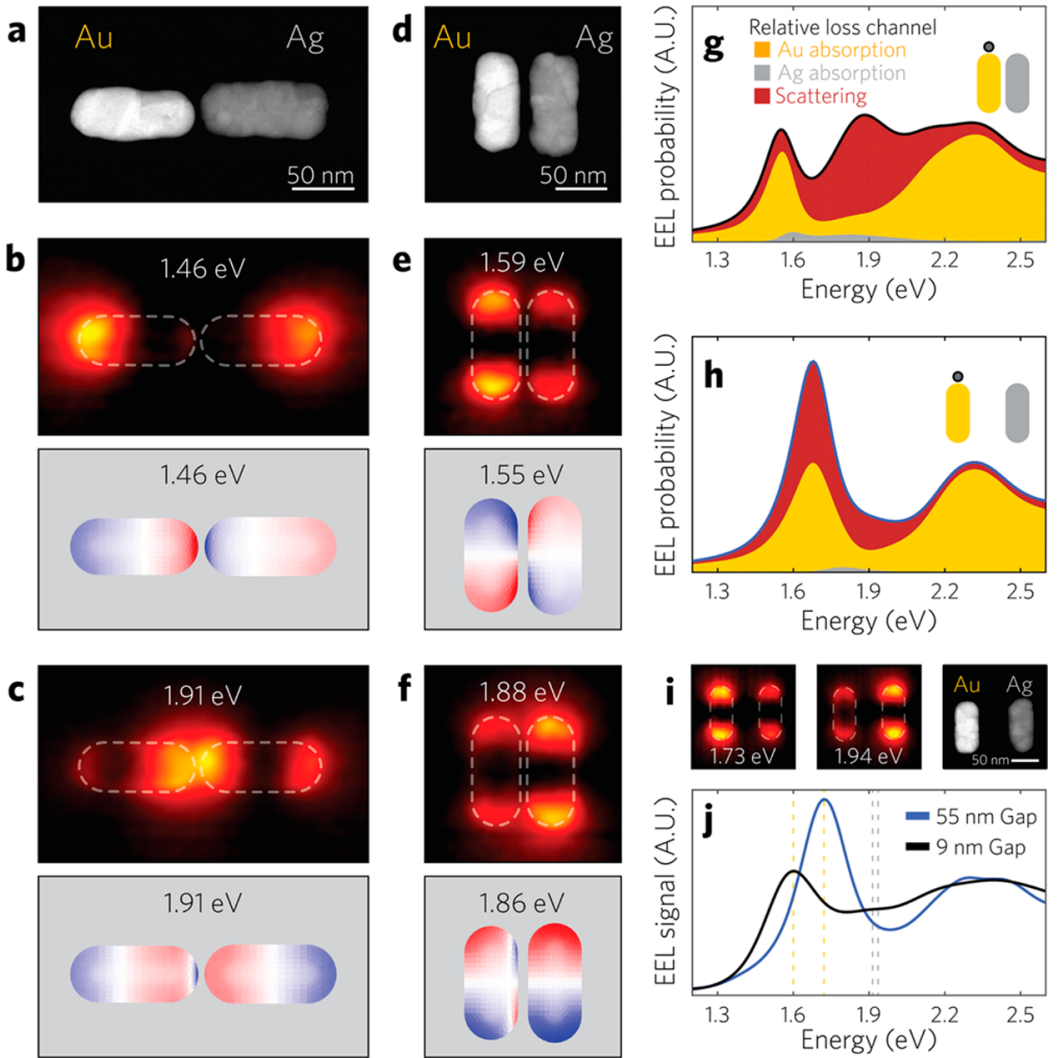

Figure 6. Nanorod heterodimers. (a) HAADF STEM image of a Au-Ag heterodimer, where the nanorods are coupled by their apexes. EELS maps and computed eigencharge distributions for (b) the bonding and (c) antibonding dipole modes. (d) HAADF STEM image of a Au-Ag heterodimer, where the nanorods are coupled by their edges. EELS maps and computed eigencharge distributions for (e) the bonding and (f) antibonding dipole modes. Computed EELS signal from the end of the Au nanorod from a parallel dimer with $(\mathrm{g})$ a $9 \mathrm{~nm}$ gap and (h) a $55 \mathrm{~nm}$ gap, respectively. The decomposition into the different loss mechanisms is represented by the filled areas under the curves. (i) EELS maps and HAADF image of the $55 \mathrm{~nm}$ gap parallel heterodimer. (j) Measured EEL spectra integrated over the Au nanorod for the two heterodimers.

charge deformations involving multiple contributions in the $\mathrm{Al}$ nanoparticle. At $2.22 \mathrm{eV}$, the quadrupole supported by the $\mathrm{Al}$ nanosquare is then imaged overlapping in energy with the breathing mode in the Au nanoparticle, with high EELS signal at the square center. ${ }^{56}$ Uncoupled high order multipoles in the $\mathrm{Al}$ square are mapped at 3.14 (octupole) and $3.80 \mathrm{eV}$, with additional contribution of a breathing mode for the latter (Figure 51). The engineering of the gap geometry therefore allows resonant coupling conditions to be achieved beyond those of the well-studied dipole-dipole interactions or dipolequadrupole interactions observed in the disc heterodimers.

Finally, in order to tune resonant coupling conditions even further, heterodimers composed of $\mathrm{Au}$ and $\mathrm{Ag}$ nanorods are considered. Contrary to the nanodiscs and nanosquares studied previously, individual nanorods possess longitudinal and transverse modes at distinct energies. When coupled, this property is useful to favor selected interactions, depending on the spatial arrangement of the nanorods. As serial (i.e., end-toend) nanorod homodimers are coupled by their apexes, the low energy bonding mode is bright and the high energy antibonding mode is dark. ${ }^{28,58}$ This relation is inverted in the case of parallel (i.e., side-by-side) rod homodimers, which are coupled by their edges. ${ }^{28,58}$ Considering these geometries in heterodimer form (Figure 6a,d), in both cases the hybridized modes observed at the lowest energy correspond to a strong excitation of the nanorod which has the lowest energy resonant dipole mode (Figure 6b,e). At the same time, the high energy mode corresponds to a strong excitation of the nanorod possessing the dipole mode resonant at the highest energy (Figure $6 \mathrm{c}, \mathrm{f}$ ). When the coupling is weak, i.e., when the gap is larger than $\sim 10 \mathrm{~nm}$, the distinct radiative signatures of both components is recognized in the global (plane wave excitation) response of the dimers, and a Fano resonance is observed due to the interference between the dipolar modes (Figure S7b). ${ }^{18,59}$ Upon increased coupling (e.g., by reducing gap size), the radiative nature of one or the other mode is significantly modulated, leading to a weakening of the Fano line shape as well as the appearance of a strong absorption modulation, as previously reported for spherical heterodimers. ${ }^{19,20,60}$ EELS does not only provide information on the near field distributions and the modal nature of the observed peaks, but also an insight into the evolution of the absorption and scattering mechanisms. ${ }^{61-63}$ Indeed, the relative weights of these two loss channels is expected to evolve upon geometric modifications. For example, in the case of the parallel rod dimers, a strong reduction of the radiative channel is seen as the gap width reduces, as emphasized by the simulated EELS decompositions (Figure $6 \mathrm{~g}, \mathrm{~h}$ and also evidenced by the plane wave scattering Figure S7b). ${ }^{64}$ This weakened scattering, responsible for the reduction of the absolute EELS signal, is well measured experimentally (Figure 6i,j and Figure S8), emphasizing that the combination of EELS with accurate modeling permits the tracking of energy loss mechanisms in heterodimers. By tuning the gap width and the parallel or serial arrangement of nanorod $\mathrm{Au}-\mathrm{Ag}$ dimers, one can therefore favor or inhibit the optical access, namely the net dipole 
moment, of modes associated with either the $\mathrm{Au}$ or $\mathrm{Ag}$ constituent. In turn, this enables a specific engineering of the absorption within the system, as in the parallel nanorod configuration where a large enhancement of absorption in the $\mathrm{Au}$ nanoparticle, at the resonance energy of the Ag nanorod, may be achieved with increased coupling.

\section{CONCLUSIONS}

In conclusion, by combining the high flexibility of electron beam lithography for the fabrication of coupled plasmonic nanostructures of various geometries with near field imaging using EELS, we have reported experimental insights into the coupling occurring in heterogeneous plasmonic dimer systems. The case of heterodimers composed of discs was considered first, and then used as a benchmark for the investigation of the role of the gap lateral extent (in square nanodimers) and mode selection (in nanorod dimers). Especially, the importance of both the spatial and spectral overlap between the modes has been explored. The results provide opportunities for the design of heterodimers with tailored interactions, such as the coupling of plasmonic modes of different multipolar nature or the engineering of absorption within the dimers. They therefore provide efficient means to control of the nanoscale optical response, enabling efficient tuning of directional scattering, ${ }^{14}$ Fano interferences, ${ }^{65}$ molecular sensing ${ }^{66}$ and multiple bright resonances for broadband fluorescence enhancement, ${ }^{67}$ as well as second harmonic generation. ${ }^{68}$

\section{METHODS}

Sample Fabrication. Silicon wafers $(100 \mathrm{~mm}$ diameter, $380 \mu \mathrm{m}$ thickness prime grade double side polished) were cleaned following a standard RCA procedure prior to the fabrication of front side alignment marks. Positive photoresist (AZ ECI 3000, $0.6 \mu \mathrm{m}$ ) was spun and exposed by direct laser writing (Heidelberg Instruments VPG-200) and developed. The markers were etched in the Si wafers by RF biased SF6 dry etching (Adixen AMS200) to a depth of $3 \mu \mathrm{m}$ followed by another RCA cleaning step and low pressure chemical vapor deposition of $30 \mathrm{~nm}$-thick silicon nitride. Backside photolithography and wet etching by potassium hydroxide were performed to release $500 \times 100 \mu \mathrm{m}^{2}$ freestanding silicon nitride windows. A single layer of poly(methyl methacrylate), (PMMA 495 A2, MicroChem) was then spun at $2500 \mathrm{rpm}$ for $60 \mathrm{~s}$ yielding an approximately $90 \mathrm{~nm}$-thick coating following a $3 \mathrm{~min}$ bake at $180{ }^{\circ} \mathrm{C}$ prior to the coating of a conductive layer (Espacer $300 \mathrm{Z}$ ShowaDenko, $2000 \mathrm{rpm}$, $60 \mathrm{~s})$. The samples were then exposed by electron beam lithography (VISTEC EBPG5000+, $100 \mathrm{kV}$ ), rinsed in deionized water to remove the conductive coating and developed at room temperature for $60 \mathrm{~s}$ in a 1:3 mixture of methyl isobutyl ketone and isopropyl alcohol. Chromium and Gold $(0.5 \mathrm{~nm} / 25 \mathrm{~nm})$ were evaporated first by electron beam heating at a base pressure of $8 \times 10^{-7} \mathrm{mBar}$ prior to liftoff in acetone. In a second step, lithography, evaporation and lift-off were repeated for the $\mathrm{Ag}$ or $\mathrm{Al}$ structures. Al was evaporated with 35 $\mathrm{nm}$ thickness after overnight pumping of the evaporation chamber to reduce residual water at the wafer surface in order to obtain low line edge roughness and high shape accuracy. Ag was evaporated with 30 $\mathrm{nm}$ thickness using a $0.5 \mathrm{~nm} \mathrm{Ni}$ wetting layer that was shown to improve the quality of otherwise poorly wetting Ag films. ${ }^{69}$ Alignment markers were protected by Kapton stencils during each evaporation steps. After all lift-off steps, the samples were finally cleaved in $3 \times 3$ $\mathrm{mm}^{2}$ dies for measurements in the TEM and stored in $\mathrm{N}_{2}$ cabinets in order to preserve Ag from ambient contamination.

Alignment Accuracy. In order to assess the accuracy and repeatability of feature dimensions and gap widths, heterodimer arrays and Vernier patterns were imaged in both TEM and STEM mode (FEI Talos $200 \mathrm{kV}$ ) and the images were processed with a custom Matlab toolbox. The Vernier patterns (S1) allowed for the measurement of alignment accuracy with limited influence of metal line edge roughness. Inspection of the Vernier patterns revealed consistent ( $90 \%)$ alignment equal or better than $10 \mathrm{~nm}$ offset on each axis. An example with sub-5 nm alignment in shown in Figure $S 1 c$,e. In addition to layer to layer alignment, metal wetting and line edge roughness were found to be the main contributions of gap variations with a standard deviation $\sim 2.5 \mathrm{~nm}$ (Figure S1f,g). Overall, designs included arrays with gaps down to $10 \mathrm{~nm}$ overlap guaranteeing that the target designs of sub-5 nm were consistently achieved.

EELS Measurements. STEM-EELS maps were acquired using a FEI Titan Themis 60-300 equipped with a Wien-type monochromator and a Gatan GIF Quantum ERS spectrometer. A $300 \mathrm{keV}$ incident electron beam was used for all experiments, monochromated to give an energy spread of $\sim 110 \mathrm{meV}$ fwhm in the zero-loss peak of elastically scattered electrons, and with beam currents of $\sim 240 \mathrm{pA}$. A $17 \mathrm{mrad}$ convergence semiangle of the probe and a $22 \mathrm{mrad}$ collection semiangle on the spectrometer were used, with the probe having a mean diameter of $<1 \mathrm{~nm}$ for full width at tenth maximum in incident intensity. Mapping was performed using the "ultrafast" spectrum imaging mode with typical dwell times of 0.20 to $0.26 \mathrm{~ms}$ per pixel, and with the probe rastered in X, Y step sizes of $0.5-0.6 \mathrm{~nm}$ for a total of $>10^{5}$ pixels per map. Each map was treated with the HQ Dark Correction plugin to reduce noise associated with dark current subtraction.

EELS Data Processing. The EELS data cubes were processed using Gatan Digital Micrograph and custom Matlab scripts for the removal of the background from the tails of the zero-loss peak (ZLP), and extraction of point spectra and spatial EELS maps. The ZLP was first centered pixel by pixel using a Gaussian-Lorentzian approximation. Following zero-loss alignment, each data cube was spectrally cropped to the region of interest including the ZLP ( -2 to $6 \mathrm{eV}$ ), and artifacts from cosmic rays were removed. To account for the absorption and other scattering mechanisms inside the metal nanoparticles, the data cubes were normalized by dividing each pixel-spectrum by the integrated zero-loss fit. Spectra in Figure 3 were integrated over a $30 \times 30$ pixel region of interest centered around the point overlaid on the STEM image, whereas EELS maps were typically integrated over a $0.06 \mathrm{eV}$ window. For visualization purposes, the maps were smoothed using a penalized least-square method. ${ }^{70}$ Overall, the resonance peak positions are identified from spectra extracted in regions with high EELS signal and with minimal spatial overlap with other resonances allowing for a high fitting accuracy, the main limitations being the effect of the precision of the ZLP alignment at higher energy losses and the ZLP convolution with the resonances at lower energy losses.

Simulations. A surface integral equation method $^{71}$ is used to compute the interaction between the electromagnetic field associated with the swift electron and the plasmonic nanostructures as well as for the computation of the eigenmodes. The nanostructure's surface is discretized with triangular mesh elements and the electric and magnetic fields in the entire space (inside and outside the nanoparticle) are related to fictitious electric and magnetic surface currents through a matrix describing the geometry and material of the structure.

EELS is computed in two steps: first by exciting the structure with the fields associated with a swift electron, and then by computing the work done on the electron by the Lorentz force against this scattered field. ${ }^{72,73}$ The eigenmodes are found by looking for the eigenvectors of the aforementioned matrix when no excitation is imposed..$^{53}$ Concerning the eigenmode analysis, a Drude model is used for the permittivities of $\mathrm{Au}, \mathrm{Ag}$ and $\mathrm{Al}$, fitted to the experimental values found in ref 74 for $\mathrm{Au}$ and $\mathrm{Ag}$ and in ref 75 for Al. The same experimental values are used for the computation of the EELS and plane wave spectra. For all simulations, a homogeneous background medium of permittivity $\varepsilon=1.8$ (index $n=1.34$ ) is used to account for the influence the $\mathrm{SiN}$ substrate $(\varepsilon=4)$. 


\section{ASSOCIATED CONTENT}

\section{S Supporting Information}

The Supporting Information is available free of charge on the ACS Publications website at DOI: 10.1021/acsnano.6b08589.

Layer to layer alignment accuracy, EDX spectra of an $\mathrm{Au}-\mathrm{Ag}$ dimer, computed eigenmodes for single and coupled $\mathrm{Au}, \mathrm{Ag}$, and $\mathrm{Al}$ discs, $\mathrm{Au}-\mathrm{Ag} 100 \mathrm{~nm}$ disc dimer EELS simulations, $\mathrm{Au}-\mathrm{Ag} 100 \mathrm{~nm}$ disc dimer multipolar decomposition, $\mathrm{Au}-\mathrm{Ag} 50 \mathrm{~nm}$ disc heterodimer, $\mathrm{Au}-\mathrm{Ag}$ rod heterodimer optical response, EEL spectra and loss channel decomposition with impact parameter on the $\mathrm{Ag}$ rod end (PDF)

\section{AUTHOR INFORMATION}

\section{Corresponding Author}

*E-mail: juergen.brugger@epfl.ch.

\section{ORCID}

Valentin Flauraud: 0000-0002-1393-3198

Jérémy Butet: 0000-0001-9598-9074

Duncan T. L. Alexander: 0000-0003-4350-8587

\section{Notes}

The authors declare no competing financial interest.

\section{ACKNOWLEDGMENTS}

The research leading to these results has received funding from the European Commission's Seventh Framework Programme (FP7-ICT-2011-7) under grant agreements 288263 (NanoVista) and ERC-2015-AdG-695206 (Nanofactory) and from the Swiss National Science Foundation (SNSF) (200020_153662). The authors gratefully acknowledge the valuable support from the EPFL center of micro and nanofabrication (CMi).

\section{REFERENCES}

(1) Biagioni, P.; Huang, J.; Hecht, B. Nanoantennas for Visible and Infrared Radiation. Rep. Prog. Phys. 2012, 75, 024402.

(2) Mühlschlegel, P.; Eisler, H.-J.; Martin, O. J. F.; Hecht, B.; Pohl, D. W. Resonant Optical Antennas. Science 2005, 308, 1607-1609.

(3) Novotny, L.; van Hulst, N. Antennas for Light. Nat. Photonics 2011, 5, 83-90.

(4) Kelly, K. L.; Coronado, E.; Zhao, L. L.; Schatz, G. C. The Optical Properties of Metal Nanoparticles: The Influence of Size, Shape, and Dielectric Environment. J. Phys. Chem. B 2003, 107, 668-677.

(5) Oldenburg, S. J.; Hale, G. D.; Jackson, J. B.; Halas, N. J. Light Scattering from Dipole and Quadrupole Nanoshell Antennas. Appl. Phys. Lett. 1999, 75, 1063-1065.

(6) Jain, P. K.; El-Sayed, M. A. Plasmonic Coupling in Noble Metal Nanostructures. Chem. Phys. Lett. 2010, 487, 153-164.

(7) Nordlander, P.; Oubre, C.; Prodan, E.; Li, K.; Stockman, M. I. Plasmon Hybridization in Nanoparticle Dimers. Nano Lett. 2004, 4, 899-903.

(8) Prodan, E.; Radloff, C.; Halas, N. J.; Nordlander, P. A Hybridization Model for the Plasmon Response of Complex Nanostructures. Science 2003, 302, 419-422.

(9) Kinkhabwala, A.; Yu, Z.; Fan, S.; Avlasevich, Y.; Mullen, K.; Moerner, W. E. Large Single-Molecule Fluorescence Enhancements Produced by a Bowtie Nanoantenna. Nat. Photonics 2009, 3, 654-657.

(10) Arroyo, J. O.; Kukura, P. Non-Fluorescent Schemes for SingleMolecule Detection, Imaging and Spectroscopy. Nat. Photonics 2016, $10,11-17$.

(11) Zhang, W.; Fischer, H.; Schmid, T.; Zenobi, R.; Martin, O. J. F. Mode-Selective Surface-Enhanced Raman Spectroscopy Using Nanofabricated Plasmonic Dipole Antennas. J. Phys. Chem. C 2009, 113, 14672-14675.
(12) Chu, M.-W.; Myroshnychenko, V.; Chen, C. H.; Deng, J.-P.; Mou, C.-Y.; García de Abajo, F. J. Probing Bright and Dark SurfacePlasmon Modes in Individual and Coupled Noble Metal Nanoparticles Using an Electron Beam. Nano Lett. 2009, 9, 399-404.

(13) Pakizeh, T.; Käll, M. Unidirectional Ultracompact Optical Nanoantennas. Nano Lett. 2009, 9, 2343-2349.

(14) Shegai, T.; Chen, S.; Miljkovic, V. D.; Zengin, G.; Johansson, P.; Kall, M. A Bimetallic Nanoantenna for Directional Colour Routing. Nat. Commun. 2011, 2, 481.

(15) Shegai, T.; Johansson, P.; Langhammer, C.; Käll, M. Directional Scattering and Hydrogen Sensing by Bimetallic Pd-Au Nanoantennas. Nano Lett. 2012, 12, 2464-2469.

(16) Brown, L. V.; Sobhani, H.; Lassiter, J. B.; Nordlander, P.; Halas, N. J. Heterodimers: Plasmonic Properties of Mismatched Nanoparticle Pairs. ACS Nano 2010, 4, 819-832.

(17) Peña-Rodríguez, O.; Pal, U.; Campoy-Quiles, M.; RodríguezFernández, L.; Garriga, M.; Alonso, M. I. Enhanced Fano Resonance in Asymmetrical Au:Ag Heterodimers. J. Phys. Chem. C 2011, 115, 64106414.

(18) Yang, Z.-J.; Zhang, Z.-S.; Zhang, W.; Hao, Z.-H.; Wang, Q.-Q. Twinned Fano Interferences Induced by Hybridized Plasmons in $\mathrm{Au}-$ Ag Nanorod Heterodimers. Appl. Phys. Lett. 2010, 96, 131113.

(19) Lombardi, A.; Grzelczak, M. P.; Pertreux, E.; Crut, A.; Maioli, P.; Pastoriza-Santos, I.; Liz-Marzán, L. M.; Vallée, F.; Del Fatti, N. Fano Interference in the Optical Absorption of an Individual Gold-Silver Nanodimer. Nano Lett. 2016, 16, 6311-6316.

(20) Bachelier, G.; Russier-Antoine, I.; Benichou, E.; Jonin, C.; Del Fatti, N.; Vallée, F.; Brevet, P. F. Fano Profiles Induced by Near-Field Coupling in Heterogeneous Dimers of Gold and Silver Nanoparticles. Phys. Rev. Lett. 2008, 101, 197401.

(21) Horneber, A.; Baudrion, A.-L.; Adam, P.-M.; Meixner, A. J.; Zhang, D. Compositional-Asymmetry Influenced Non-Linear Optical Processes of Plasmonic Nanoparticle Dimers. Phys. Chem. Chem. Phys. 2013, 15, 8031-8034.

(22) Toroghi, S.; Kik, P. G. Photothermal Response Enhancement in Heterogeneous Plasmon-Resonant Nanoparticle Trimers. Phys. Rev. B: Condens. Matter Mater. Phys. 2014, 90, 205414.

(23) Evlyukhin, A. B.; Bozhevolnyi, S. I.; Pors, A.; Nielsen, M. G.; Radko, I. P.; Willatzen, M.; Albrektsen, O. Detuned Electrical Dipoles for Plasmonic Sensing. Nano Lett. 2010, 10, 4571-4577.

(24) Gschneidtner, T. A.; Fernandez, Y. A. D.; Syrenova, S.; Westerlund, F.; Langhammer, C.; Moth-Poulsen, K. A Versatile SelfAssembly Strategy for the Synthesis of Shape-Selected Colloidal Noble Metal Nanoparticle Heterodimers. Langmuir 2014, 30, 3041-3050.

(25) Liu, N.; Tang, M. L.; Hentschel, M.; Giessen, H.; Alivisatos, A. P. Nanoantenna-Enhanced Gas Sensing in a Single Tailored Nanofocus. Nat. Mater. 2011, 10, 631-636.

(26) Swearer, D. F.; Zhao, H.; Zhou, L.; Zhang, C.; Robatjazi, H.; Martirez, J. M. P.; Krauter, C. M.; Yazdi, S.; McClain, M. J.; Ringe, E.; Carter, E. A.; Nordlander, P.; Halas, N. J. Heterometallic AntennaReactor Complexes for Photocatalysis. Proc. Natl. Acad. Sci. U. S. A. 2016, 113, 8916-8920.

(27) Zhang, C.; Zhao, H.; Zhou, L.; Schlather, A. E.; Dong, L.; McClain, M. J.; Swearer, D. F.; Nordlander, P.; Halas, N. J. Al-Pd Nanodisk Heterodimers as Antenna-Reactor Photocatalysts. Nano Lett. 2016, 16, 6677-6682.

(28) Slaughter, L. S.; Wu, Y.; Willingham, B. A.; Nordlander, P.; Link, S. Effects of Symmetry Breaking and Conductive Contact on the Plasmon Coupling in Gold Nanorod Dimers. ACS Nano 2010, 4, 4657-4666.

(29) Sheikholeslami, S.; Jun, Y.-w.; Jain, P. K.; Alivisatos, A. P. Coupling of Optical Resonances in a Compositionally Asymmetric Plasmonic Nanoparticle Dimer. Nano Lett. 2010, 10, 2655-2660.

(30) Weller, L.; Thacker, V. V.; Herrmann, L. O.; Hemmig, E. A.; Lombardi, A.; Keyser, U. F.; Baumberg, J. J. Gap-Dependent Coupling of $\mathrm{Ag}-\mathrm{Au}$ Nanoparticle Heterodimers Using DNA Origami-Based Self-Assembly. ACS Photonics 2016, 3, 1589-1595.

(31) Schubert, I.; Sigle, W.; van Aken, P. A.; Trautmann, C.; ToimilMolares, M. E. STEM-EELS Analysis of Multipole Surface Plasmon 
Modes in Symmetry-Broken AuAg Nanowire Dimers. Nanoscale 2015, 7, 4935-4941.

(32) Zhaogang, D.; Michel, B.; Di, Z.; Xiao Ming, G.; Joel, K. W. Y. Fabrication of Suspended Metal-Dielectric-Metal Plasmonic Nanostructures. Nanotechnology 2014, 25, 135303.

(33) Zhang, M.; Large, N.; Koh, A. L.; Cao, Y.; Manjavacas, A.; Sinclair, R.; Nordlander, P.; Wang, S. X. High-Density 2D Homo- and Hetero- Plasmonic Dimers with Universal Sub-10-nm Gaps. ACS Nano 2015, 9, 9331-9339.

(34) Greybush, N. J.; Saboktakin, M.; Ye, X.; Della Giovampaola, C.; Oh, S. J.; Berry, N. E.; Engheta, N.; Murray, C. B.; Kagan, C. R. Plasmon-Enhanced Upconversion Luminescence in Single Nanophosphor-Nanorod Heterodimers Formed through Template-Assisted Self-Assembly. ACS Nano 2014, 8, 9482-9491.

(35) Flauraud, V.; Mastrangeli, M.; Bernasconi, G. D.; Butet, J.; Alexander, D. T.; Shahrabi, E.; Martin, O. J.; Brugger, J. Nanoscale Topographical Control of Capillary Assembly of Nanoparticles. Nat. Nanotechnol. 2017, 12, 73-80.

(36) Diaz Fernandez, Y. A.; Gschneidtner, T. A.; Wadell, C.; Fornander, L. H.; Lara Avila, S.; Langhammer, C.; Westerlund, F.; Moth-Poulsen, K. The Conquest of Middle-Earth: Combining TopDown and Bottom-Up Nanofabrication for Constructing Nanoparticle Based Devices. Nanoscale 2014, 6, 14605-14616.

(37) Goeken, K. L.; Subramaniam, V.; Gill, R. Enhancing Spectral Shifts of Plasmon-Coupled Noble Metal Nanoparticles for Sensing Applications. Phys. Chem. Chem. Phys. 2015, 17, 422-427.

(38) Romo-Herrera, J. M.; Alvarez-Puebla, R. A.; Liz-Marzan, L. M. Controlled Assembly of Plasmonic Colloidal Nanoparticle Clusters. Nanoscale 2011, 3, 1304-1315.

(39) Wersäll, M.; Verre, R.; Svedendahl, M.; Johansson, P.; Käll, M.; Shegai, T. Directional Nanoplasmonic Antennas for Self-Referenced Refractometric Molecular Analysis. J. Phys. Chem. C 2014, 118, 21075-21080.

(40) Wadell, C.; Langhammer, C. Drift-Corrected Nanoplasmonic Hydrogen Sensing by Polarization. Nanoscale 2015, 7, 10963-10969.

(41) Yang, A.; Huntington, M. D.; Cardinal, M. F.; Masango, S. S.; Van Duyne, R. P.; Odom, T. W. Hetero-Oligomer Nanoparticle Arrays for Plasmon-Enhanced Hydrogen Sensing. ACS Nano 2014, 8, 76397647.

(42) Syrenova, S.; Wadell, C.; Langhammer, C. Shrinking-Hole Colloidal Lithography: Self-Aligned Nanofabrication of Complex Plasmonic Nanoantennas. Nano Lett. 2014, 14, 2655-2663.

(43) Nugroho, F. A. A.; Iandolo, B.; Wagner, J. B.; Langhammer, C. Bottom-Up Nanofabrication of Supported Noble Metal Alloy Nanoparticle Arrays for Plasmonics. ACS Nano 2016, 10, 2871-2879.

(44) Guisbiers, G.; Mendoza-Cruz, R.; Bazán-Díaz, L.; VelázquezSalazar, J. J.; Mendoza-Perez, R.; Robledo-Torres, J. A.; RodriguezLopez, J.-L.; Montejano-Carrizales, J. M.; Whetten, R. L.; JoséYacamán, M. Electrum, the Gold-Silver Alloy, from the Bulk Scale to the Nanoscale: Synthesis, Properties, and Segregation Rules. ACS Nano 2016, 10, 188-198.

(45) Zorić, I.; Zäch, M.; Kasemo, B.; Langhammer, C. Gold, Platinum, and Aluminum Nanodisk Plasmons: Material Independence, Subradiance, and Damping Mechanisms. ACS Nano 2011, 5, 25352546.

(46) McPeak, K. M.; Jayanti, S. V.; Kress, S. J. P.; Meyer, S.; Iotti, S.; Rossinelli, A.; Norris, D. J. Plasmonic Films Can Easily Be Better: Rules and Recipes. ACS Photonics 2015, 2, 326-333.

(47) Knight, M. W.; King, N. S.; Liu, L.; Everitt, H. O.; Nordlander, P.; Halas, N. J. Aluminum for Plasmonics. ACS Nano 2014, 8, 834840.

(48) Ordal, M. A.; Long, L. L.; Bell, R. J.; Bell, S. E.; Bell, R. R.; Alexander, R. W.; Ward, C. A. Optical Properties of the Petals Al, Co, $\mathrm{Cu}, \mathrm{Au}, \mathrm{Fe}, \mathrm{Pb}, \mathrm{Ni}, \mathrm{Pd}, \mathrm{Pt}, \mathrm{Ag}, \mathrm{Ti}$, and $\mathrm{W}$ in the Infrared and Far Infrared. Appl. Opt. 1983, 22, 1099-1119.

(49) Rossouw, D.; Couillard, M.; Vickery, J.; Kumacheva, E.; Botton, G. A. Multipolar Plasmonic Resonances in Silver Nanowire Antennas Imaged with a Subnanometer Electron Probe. Nano Lett. 2011, 11, 1499-1504.
(50) Duan, H.; Fernández-Domínguez, A. I.; Bosman, M.; Maier, S. A.; Yang, J. K. W. Nanoplasmonics: Classical down to the Nanometer Scale. Nano Lett. 2012, 12, 1683-1689.

(51) Haberfehlner, G.; Trügler, A.; Schmidt, F. P.; Hörl, A.; Hofer, F.; Hohenester, U.; Kothleitner, G. Correlated 3D Nanoscale Mapping and Simulation of Coupled Plasmonic Nanoparticles. Nano Lett. 2015, $15,7726-7730$.

(52) Flauraud, V.; Regmi, R.; Winkler, P. M.; Alexander, D. T. L.; Rigneault, H.; van Hulst, N. F.; García-Parajo, M. F.; Wenger, J.; Brugger, J. In-Plane Plasmonic Antenna Arrays with Surface Nanogaps for Giant Fluorescence Enhancement. Nano Lett. 2017, 17, 17031710.

(53) Bernasconi, G. D.; Butet, J.; Martin, O. J. F. Mode Analysis of Second-Harmonic Generation in Plasmonic Nanostructures. J. Opt. Soc. Am. B 2016, 33, 768-779.

(54) Hörl, A.; Trügler, A.; Hohenester, U. Tomography of Particle Plasmon Fields from Electron Energy Loss Spectroscopy. Phys. Rev. Lett. 2013, 111, 076801.

(55) Hörl, A.; Trügler, A.; Hohenester, U. Full Three-Dimensonal Reconstruction of the Dyadic Green Tensor from Electron Energy Loss Spectroscopy of Plasmonic Nanoparticles. ACS Photonics 2015, 2, 1429-1435.

(56) Schmidt, F.-P.; Ditlbacher, H.; Hohenester, U.; Hohenau, A.; Hofer, F.; Krenn, J. R. Dark Plasmonic Breathing Modes in Silver Nanodisks. Nano Lett. 2012, 12, 5780-5783.

(57) Quillin, S. C.; Cherqui, C.; Montoni, N. P.; Li, G.; Camden, J. P.; Masiello, D. J. Imaging Plasmon Hybridization in Metal Nanoparticle Aggregates with Electron Energy-Loss Spectroscopy. J. Phys. Chem. C 2016, 120, 20852-20859.

(58) Kern, J.; Großmann, S.; Tarakina, N. V.; Häckel, T.; Emmerling, M.; Kamp, M.; Huang, J.-S.; Biagioni, P.; Prangsma, J. C.; Hecht, B. Atomic-Scale Confinement of Resonant Optical Fields. Nano Lett. 2012, 12, 5504-5509.

(59) Lovera, A.; Gallinet, B.; Nordlander, P.; Martin, O. J. F. Mechanisms of Fano Resonances in Coupled Plasmonic Systems. ACS Nano 2013, 7, 4527-4536.

(60) Lombardi, A.; Grzelczak, M. P.; Crut, A.; Maioli, P.; PastorizaSantos, I.; Liz-Marzán, L. M.; Del Fatti, N.; Vallée, F. Optical Response of Individual Au-Ag@SiO2 Heterodimers. ACS Nano 2013, 7, 25222531.

(61) Myroshnychenko, V.; Nelayah, J.; Adamo, G.; Geuquet, N.; Rodríguez-Fernández, J.; Pastoriza-Santos, I.; MacDonald, K. F.; Henrard, L.; Liz-Marzán, L. M.; Zheludev, N. I.; et al. Plasmon Spectroscopy and Imaging of Individual Gold Nanodecahedra: A Combined Optical Microscopy, Cathodoluminescence, and Electron Energy-Loss Spectroscopy Study. Nano Lett. 2012, 12, 4172-4180.

(62) Losquin, A.; Kociak, M. Link between Cathodoluminescence and Electron Energy Loss Spectroscopy and the Radiative and Full Electromagnetic Local Density of States. ACS Photonics 2015, 2, $1619-1627$.

(63) Kawasaki, N.; Meuret, S.; Weil, R; Lourenço-Martins, H.; Stéphan, O.; Kociak, M. Extinction and Scattering Properties of HighOrder Surface Plasmon Modes in Silver Nanoparticles Probed by Combined Spatially Resolved Electron Energy Loss Spectroscopy and Cathodoluminescence. ACS Photonics 2016, 3, 1654-1661.

(64) Bernasconi, G. D.; Butet, J.; Flauraud, V.; Alexander, D.; Brugger, J.; Martin, O. J. F. Where Does Energy Go in Electron Energy Loss Spectroscopy of Nanostructures? ACS Photonics 2017, 4, 156164

(65) Luk'yanchuk, B.; Zheludev, N. I.; Maier, S. A.; Halas, N. J.; Nordlander, P.; Giessen, H.; Chong, C. T. The Fano Resonance in Plasmonic Nanostructures and Metamaterials. Nat. Mater. 2010, 9, 707-715.

(66) Duan, X.; Kamin, S.; Sterl, F.; Giessen, H.; Liu, N. HydrogenRegulated Chiral Nanoplasmonics. Nano Lett. 2016, 16, 1462-1466.

(67) Mivelle, M.; van Zanten, T. S.; Garcia-Parajo, M. F. Hybrid Photonic Antennas for Subnanometer Multicolor Localization and Nanoimaging of Single Molecules. Nano Lett. 2014, 14, 4895-4900. 
(68) Butet, J.; Brevet, P.-F.; Martin, O. J. F. Optical Second Harmonic Generation in Plasmonic Nanostructures: From Fundamental Principles to Advanced Applications. ACS Nano 2015, 9, $10545-10562$.

(69) Liu, H.; Wang, B.; Leong, E. S. P.; Yang, P.; Zong, Y.; Si, G.; Teng, J.; Maier, S. A. Enhanced Surface Plasmon Resonance on a Smooth Silver Film with a Seed Growth Layer. ACS Nano 2010, 4, 3139-3146.

(70) Garcia, D. Robust Smoothing of Gridded Data in One and Higher Dimensions with Missing Values. Comput. Stat. Data Anal. 2010, 54, 1167-1178.

(71) Kern, A. M.; Martin, O. J. F. Surface Integral Formulation for 3D Simulations of Plasmonic and High Permittivity Nanostructures. J. Opt. Soc. Am. A 2009, 26, 732-740.

(72) Ritchie, R. H. Plasma Losses by Fast Electrons in Thin Films. Phys. Rev. 1957, 106, 874-881.

(73) García de Abajo, F. J. Optical Excitations in Electron Microscopy. Rev. Mod. Phys. 2010, 82, 209-275.

(74) Johnson, P. B.; Christy, R. W. Optical Constants of the Noble Metals. Phys. Rev. B 1972, 6, 4370-4379.

(75) Palik, E. D. G. Handbook of Optical Constants of Solids; Academic Press, 1985. 